PROCEEDINGS OF THE

AMERICAN MATHEMATICAL SOCIETY

Volume 125, Number 2, February 1997, Pages 625-628

S 0002-9939(97)03604-6

\title{
A NOTE ON A HOMOLOGY SPHERE
}

\author{
S. AKBULUT
}

(Communicated by Ronald Stern)

\begin{abstract}
Here we give a solution to a problem of Y.Matsumoto which was posed in "Kirby's problem list"
\end{abstract}

In this note we solve a problem posed by Y.Matsumoto in Kirby's problem list (Problem $4.28(\mathrm{~A})$ of $[\mathrm{K}]$ ). This problem should have been solved ten years ago after Donaldson's Theorem-C in $[\mathrm{D}]$ which imposed a restriction to intersection forms of certain 4 manifolds because the solution does not use any 4-manifold techniques developed since then. The problem is whether the 4-manifold $M$ obtained by attaching a pair of two handles to a 4-ball $B^{4}$ along the two linked left handed trefoil knots, as in Figure 1, contains a smoothly imbedded wedge of 2-spheres representing generators of $H_{2}(M)$ ? An affirmative answer implies that $\partial M$ bounds a contractible manifold $W$. We show that this is not the case. In fact $\partial M$ does not bound a 4-homology ball $W$ with $\pi_{1}(\partial M) \rightarrow \pi_{1}(W)$ onto.

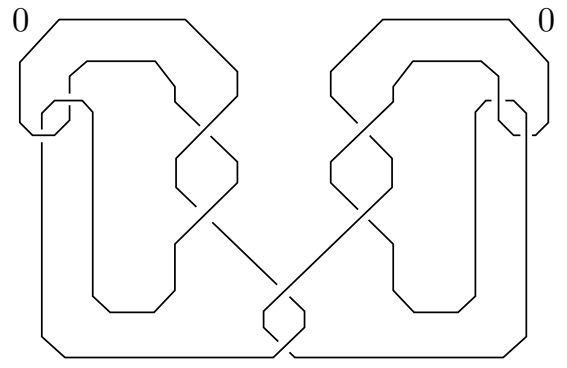

\section{FIGURE 1}

The 4-manifolds represented by Figures 1, 2, 3, 4 have the same boundary: By blowing down the $(+1)$-framed 2-handle in Figure 4 we get Figure 3; Figure 3 is obtained from Figure 2 by sliding the $(-1)$-framed handle over one of the 0 -framed handles as indicated by the dotted arrow, and then cancelling the 0 -framed handle pair; Figure 1 is obtained from Figure 2 by blowing down the two $(-1)$-framed 2-handles. Now by the usual "blowing up and down" process (e.g. [A] figures 9-19) we can turn two (-1)-framed trefoil knots of Figure 4 into two $E_{8}$ 's and obtain Figure 5. This process turns the $(+1)$-framed 2-handle into a connected sum of two right-handed trefoil knots. By introducing two hyperbolic pairs as in Figure 6 we can make the $(+1)$-framed knot of Figure 5 slice, which we can blow down.

Received by the editors September 1, 1995.

1991 Mathematics Subject Classification. Primary 57M25, 57R95; Secondary 57R65. 


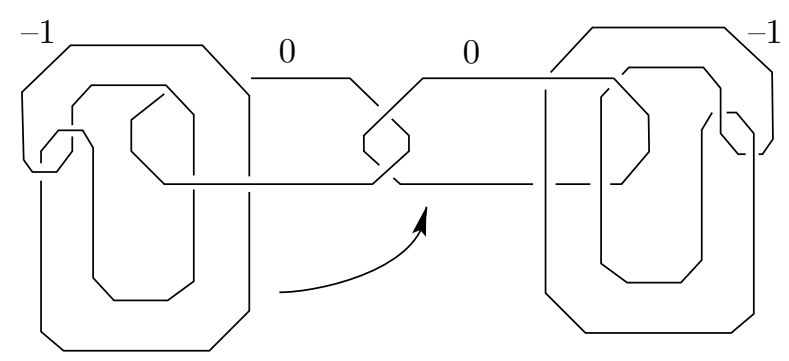

FiguRE 2

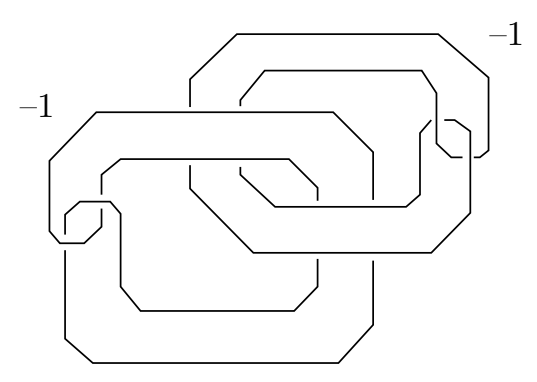

FIGURE 3

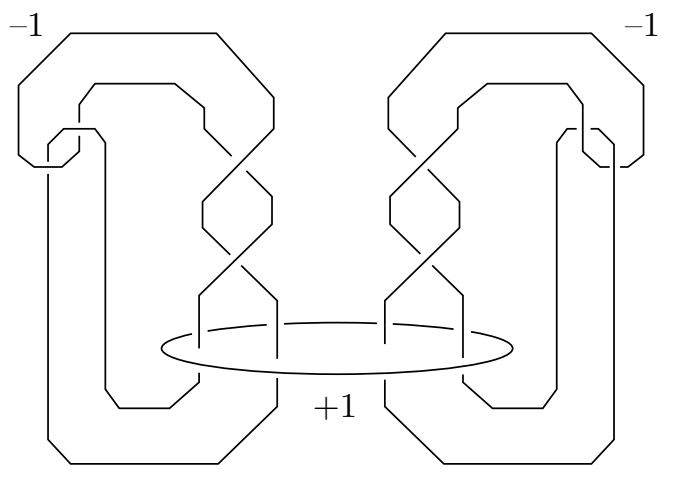

FiguRE 4

This gives a smooth spin manifold $Q$ with intersection form $E_{8} \oplus E_{8} \oplus 2\left(\begin{array}{cc}0 & 1 \\ 1 & 0\end{array}\right)$, and $\partial Q=\partial M$. Hence if $\partial M$ were to bound a contractible manifold $\mathrm{W}$, then $Q \smile(-W)$ would be a manifold violating Donaldson's Theorem-C in [D].

An interesting fact: by blowing down one of the -1 spheres of Figure 3 we see that the manifold $\partial M$ is also obtained by -1 surgery to 0 -double of the left handed trefoil knot (Figure 7). Another interesting side fact which the reader can check is that the manifold $M$ is a 2-fold branched covering of the cusp manifold (i.e. 4-ball with a 2-handle attached along the left handed trefoil knot with either $( \pm 1)$-framing, e.g. Figure 8) along a properly imbedded 2-disc (the obvious disc bounded by $\gamma$ in Figure 8). 
A NOTE ON A HOMOLOGY SPHERE

627

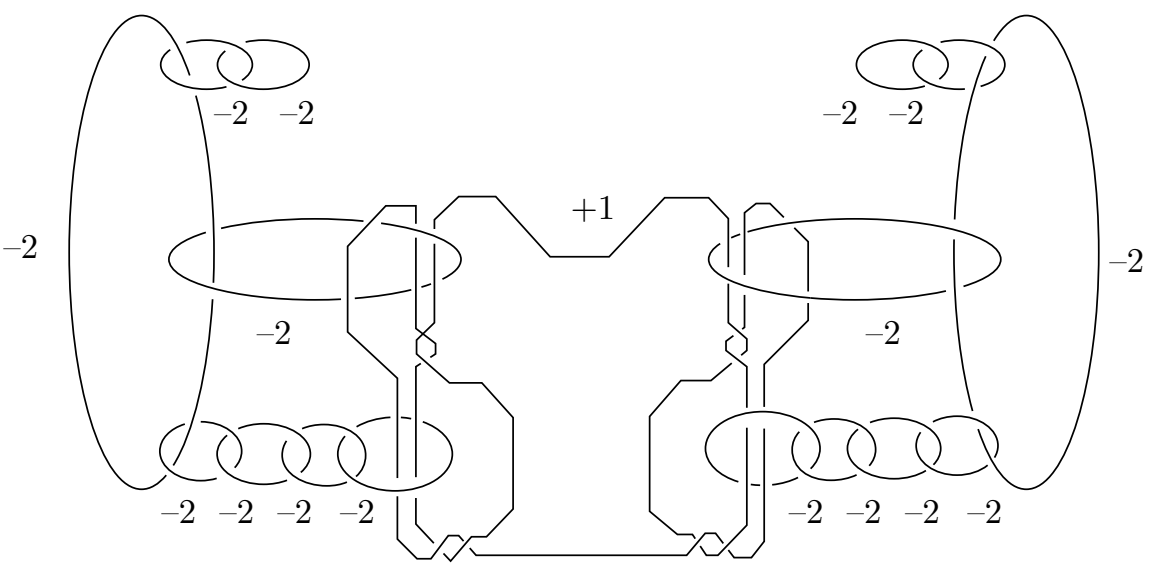

FigURE 5

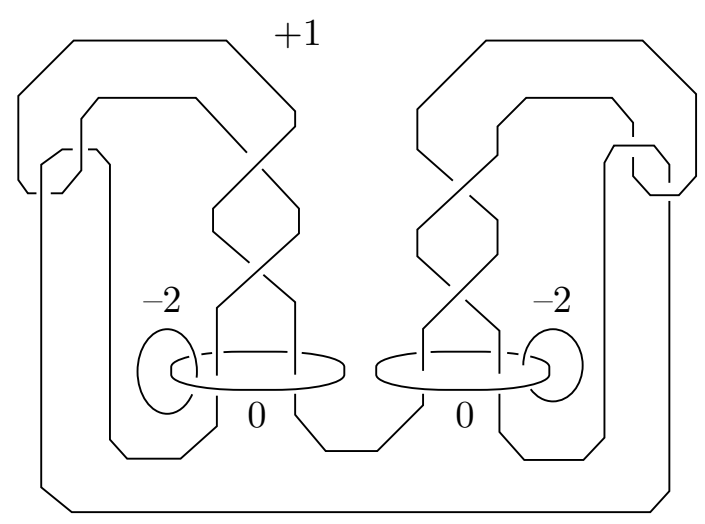

Figure 6

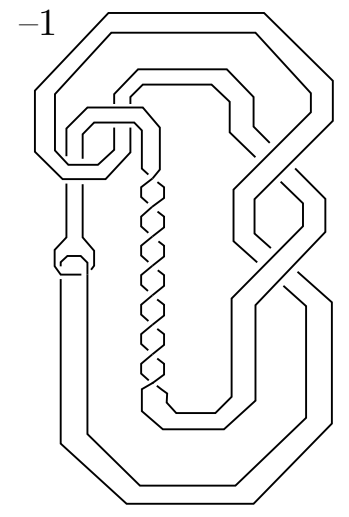

FiguRE 7 


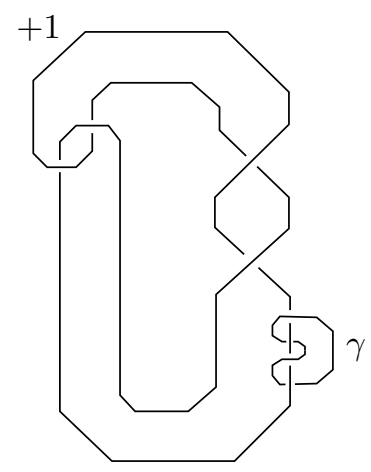

Figure 8

\section{REFERENCES}

[A] S. Akbulut, A Fake Compact Contractible 4-Manifold, Jour.Diff.Geom. 33 (1991), 335356. MR 92b:57025

[D] S. Donaldson, Connections, Cohomology and the Intersection Forms of 4-Manifolds, Jour.Diff.Geom. 24 (1986), 275-341. MR 88g:57033

[K] Rob Kirby (compiler), Problems in Low Dimensional Manifold Theory, Proceedings of Symposia in Pure Mathematics, Vol.32, (1978), 273-312. MR 80g:57002

Department of Mathematics, Michigan State University, East Lansing, Michigan 48824

E-mail address: akbulut@math.msu.edu 